\title{
Price decision making in a centralized/decentralized solid waste disposal supply chain with one contractor and two disposal facilities
}

\author{
Iman Ghalehkhondabi ${ }^{1}$ and Reza Maihami ${ }^{2}$ \\ ${ }^{1}$ School of Business and Leadership, Our Lady of the Lake University, San Antonio TX 78207, USA \\ ighalehkhonda@ollusa.edu \\ ${ }^{2}$ School of Business and Leadership, Our Lady of the Lake University, Houston TX 77067, USA \\ rmaihami@ollusa.edu
}

\begin{abstract}
Solid waste management has been an interesting topic for researchers in few last decades. This paper studies a price-sensitive demand for the waste disposal service of two disposal facilities who are dealing with a contractor to gain more profit. The waste disposal process is studied in a supply chain structure where a contractor manages to collect the waste from the producers and transport them to the facilities for disposal. Two scenarios are proposed where at the first one the disposal facilities lead a price Stackelberg game over the contractor, and in the second scenario, both disposal facilities and the contractor cooperate on the chain decision variables in an integrated framework. A numerical example is performed to illustrate the efficiency and applications of the proposed model.
\end{abstract}

Keywords: Stackelberg Game, Coordinated Supply Chain, Solid Waste Management, Pricing

\section{Introduction}

Municipal solid waste includes the produced waste by residential and business buildings, public parks, municipals, and service providers. The municipal solid waste is generally categorized to recyclables, hazardous, and garbage waste [1]. It is a challenge for many municipals to design or select a solid waste management system that minimizes the waste negative environmental impacts and ensures a reasonable level of health and welfare for the citizens [2].

Solid waste management includes managing waste generation, collection, separation, transportation, treatment, distribution and disposal, therefore we can define waste management as a supply chain management problem [3]. Having an inappropriate waste management system can lead to disease transmission, contaminated ground and surface water, negative impacts on ecosystem, producing greenhouse emissions, and negative impacts on tourism and the other business activities [4].

There are some operations research capabilities which motivate using mathematical models in solid waste management area. For instance, the hidden relation between different parameters in a problem become noticeable when developing a mathematical model. Or having the mathematical model enables the decision makers to test different scenarios and make informed decisions [5]. 
The environmental effects of solid waste management have been an important place of academic discussions in last two decades. Assessment of hospital biomedical waste management [6], relations between solid waste management and climate change [7], and sustainable design of a waste management system [3] are among some of the recent studies in this area. Besides the environmental impacts, economics always play an important role in municipal services. Pricing of the waste management service has been studied by many researchers. But, most of the waste management pricing studies consider the elasticity of waste production versus waste management service price rather than studying the profitability of the waste disposal facilities as economic entities. Weight-based pricing in the collection of household waste [9], effects of unitbased pricing on household waste collection demand [10], public willingness to pay and participate in waste management [11] are among the works which studied pricing in waste management systems.

In this study, we consider a solid waste disposal supply chain including two disposal facilities who compete for selling the service, and one contractor who is the dealer between the municipal waste producers and the disposal facilities. Demand for each disposal facility is a function of its own price and the competitor price. We solve the model under two scenarios. At the first scenario we consider a Stackelberg game, where the disposal facilities determine their service prices for the final customer (waste producer), and the contractor determines its own profit based on the given service prices. In the second scenario, an integrated supply chain is considered where one individual makes decisions for entire entities. So, the service price and the contractor profit margin are determined at the same time by one agent. Figure 1 shows the structure of this model.

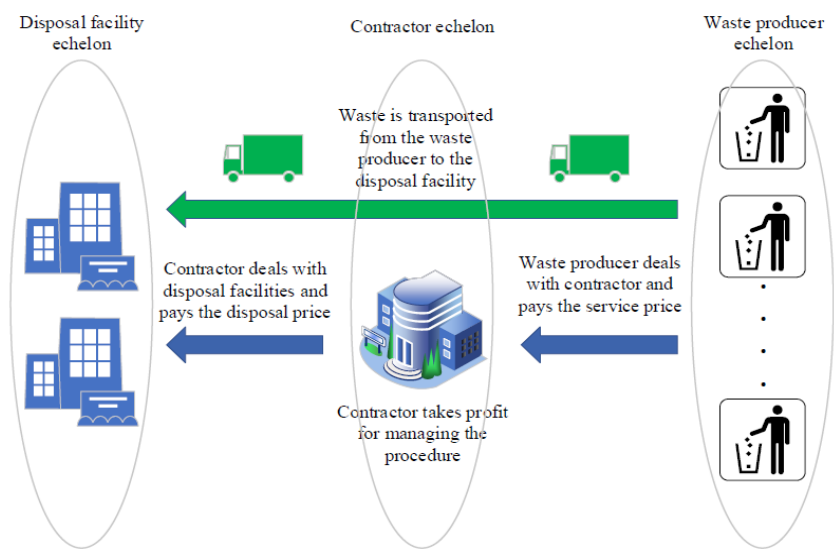

Fig. 1. Waste disposal supply chain

The objective of this study is to show the effect of integrated decision making on the decision variables and profit of a waste disposal supply chain.

\section{Model Representation}

Following notation is used in modeling the problem: 
$D_{i}$ : Demand for the service of facility $\mathrm{i}$

$d$ : The total potential demand for solid waste disposal

$p_{i}$ : Customer price for the service provided by facility i (per kilogram)

$\alpha$ : The price sensitivity of demand for facility i based on its own price

$\beta$ : The price sensitivity of demand for facility i based on its competitor price

$h_{i}$ : Disposal facility i price asked from the contractor (per kilogram)

$\mu_{i}$ : Contractor's profit for selling the service of facility i (per kilogram)

$o_{i}$ : Operation cost at facility i (per kilogram)

We observe the demand for urban solid waste disposal to be a function of price. Increasing the price in disposal facility 1 (or decreasing price in facility 2 ) may reduce the demand for this facility (Choi, 1991). Equation 1 denotes the price sensitive demand function for solid waste disposal facilities:

$D_{i}=d-\alpha p_{i}+\beta p_{3-i}, \quad i=1,2, \quad d, \alpha, \beta>0$

According to (Jeuland and Shugan, 1983), $\alpha>\beta$ should hold true, and the larger difference between these two parameters mean that two products have lower possibility of substitution.

\subsection{Decentralized waste management supply chain}

Considering the demand function (1), we can define the disposal facilities' and contractor's profit functions $\rho_{F_{i}}$ and $\rho_{C O}$ as follow:

$\rho_{F_{i}}=\left(h_{i}-o_{i}\right)\left(d-\alpha p_{i}+\beta p_{3-i}\right) \quad i=1,2$

$\rho_{C O}=\sum_{i=1}^{2} \mu_{i}\left(d-\alpha p_{i}+\beta p_{3-i}\right)$

The profit that the contractor makes on selling the service of each facility equals to the difference between the price that it receives from the customers and the cost it pays to that facility. In our first scenario we believe that the disposal facilities have enough power to make their price decisions $h_{i}$ and dictate it to the contractor. Considering the value of $h_{i}$, contractor should determine the final price asked from the customer $p_{i}$. If we substitute $\mu_{i}=p_{i}-h_{i}$, the contractor's reaction function to the prices $h_{1}$ and $h_{2}$ can be calculated through the first order derivatives of (3):

$\frac{\partial \rho_{C O}}{\partial p_{1}}=d-2 \alpha p_{1}+2 \beta p_{2}+\alpha h_{1}-\beta h_{2}$

$\frac{\partial \rho_{C O}}{\partial p_{2}}=d-2 \alpha p_{2}+2 \beta p_{1}+\alpha h_{2}-\beta h_{1}$

The coordinator profit function would be jointly concave in $p_{1}$ and $p_{2}$ if the Hessian matrix (6) is negative-definite:

$\left[\begin{array}{ll}\frac{\partial^{2} \rho_{C O}}{\partial p_{1}{ }^{2}} & \frac{\partial^{2} \rho_{C O}}{\partial p_{1} \partial p_{2}} \\ \frac{\partial^{2} \rho_{C O}}{\partial p_{1} \partial p_{2}} & \frac{\partial^{2} \rho_{C O}}{\partial p_{2}{ }^{2}}\end{array}\right]=\left[\begin{array}{cc}-2 \alpha & 2 \beta \\ 2 \beta & -2 \alpha\end{array}\right]=4 \alpha^{2}-4 \beta^{2}$

According to our assumption of $\alpha>\beta, 4 \alpha^{2}-4 \beta^{2}$ would be positive, and the Hessian matrix is negative definite. If we solve equations (4) and (5) for zero, the optimal prices are: 
$p_{1}=\frac{h_{1}}{2}+\frac{d}{2(\alpha-\beta)}$

$p_{2}=\frac{h_{2}}{2}+\frac{d}{2(\alpha-\beta)}$

Substituting the optimal price values to equation (2), the first order derivative of (2) with respect to $h_{i}$ can give us the maximization condition of the disposal facilities' profit functions:

$\frac{\partial \rho_{F_{i}}}{\partial h_{i}}=\frac{1}{2}\left(d-2 \alpha h_{i}+\beta h_{3-i}+\alpha o_{i}\right), \quad i=1,2$

Solving equation (9) for zero gives us the optimal $h_{i}$ values:

$h_{i}=\frac{d}{2(\alpha-\beta)}+\frac{\alpha\left(2 \alpha o_{i}+\beta o_{3-i}\right)}{(2 \alpha-\beta)(2 \alpha+\beta)}, \quad i=1,2$

Substituting (9) in (7), we have the optimal price asked from the customers:

$p_{i}=\frac{d(3 \alpha-2 \beta)}{2(2 \alpha-\beta)(\alpha-\beta)}+\frac{\alpha\left(2 \alpha o_{i}+\beta o_{3-i}\right)}{2(2 \alpha-\beta)(2 \alpha+\beta)}, \quad i=1,2$

To simplify the problem, we logically can assume that the operations cost for both disposal facilities is the same. This assumption also removes our analysis dependence on the used technology in each facility. Therefore, using o as the operations cost in both facilities, we have the optimal $h_{i}$ and $p_{i}$ prices as follows:

$h_{i}=\frac{d+\alpha o}{2 \alpha-\beta}, \quad i=1,2$

$p_{i}=\frac{d(3 \alpha-2 \beta)+\alpha o(\alpha-\beta)}{2(2 \alpha-\beta)(\alpha-\beta)}, \quad i=1,2$

Important model outputs can be derived using values in (12) and (13) as shown in Table 1. It is notable that the disposal facility may only cover its costs if its service price asked from the contractor is greater than or equal to the operations cost, therefore (14) should always hold true:

$\frac{d+\alpha o}{2 \alpha-\beta} \geq o \rightarrow o \leq \frac{d}{\alpha-\beta}$

\subsection{Integrated waste management supply chain}

Our second scenario considers a case where an individual agent makes the decisions for all players in the solid waste disposal chain. In this case we only have one profit function which is the supply chain profit function:

$\max _{\mu_{i}, \mu_{3-i}} \rho_{S C}=\sum_{i=1}^{2} \mu_{i}\left(d-\alpha\left(o+\mu_{i}\right)+\beta\left(o+\mu_{3-i}\right)\right)$

Model optimal values can be derived through the optimal value of $\mu_{i}$. The first order conditions with respect to $\mu_{i}$ follow:

$\frac{\partial \rho_{S C}}{\partial \mu_{1}}=d-\alpha\left(o+2 \mu_{1}\right)+\beta o+2 \beta \mu_{2}$

$\frac{\partial \rho_{S C}}{\partial \mu_{2}}=d-\alpha\left(o+2 \mu_{2}\right)+\beta o+2 \beta \mu_{1}$

The Hessian matrix is: 
$\left[\begin{array}{cc}\frac{\partial^{2} \rho_{S C}}{\partial \mu_{1}^{2}} & \frac{\partial^{2} \rho_{S C}}{\partial \mu_{1} \partial \mu_{2}} \\ \frac{\partial^{2} \rho_{S C}}{\partial \mu_{1} \partial \mu_{2}} & \frac{\partial^{2} \rho_{S C}}{\partial \mu_{2}{ }^{2}}\end{array}\right]=\left[\begin{array}{cc}-2 \alpha & 2 \beta \\ 2 \beta & -2 \alpha\end{array}\right]=4 \alpha^{2}-4 \beta^{2}$

The solid waste disposal supply chain has a maximum value for $\alpha>\beta$. Solving (16) and (17) for zero, we have:

$\mu_{1}=\frac{d-o \alpha+o \beta+2 \beta \mu_{2}}{2 \alpha}$

$\mu_{2}=\frac{d-o \alpha+o \beta+2 \beta \mu_{1}}{2 \alpha}$

Substituting (19) to (18), we have:

$\mu_{1}=\mu_{2}=\frac{d-o \alpha+o \beta}{2 \alpha-2 \beta}$

Table 1. Optimal prices and profits

\begin{tabular}{c|cc} 
Equil. value & Decentralized chain & Integrated chain \\
\hline$h_{i}$ & $\frac{d+\alpha o}{2 \alpha-\beta}$ & - \\
$p_{i}$ & $\frac{d(3 \alpha-2 \beta)+\alpha o(\alpha-\beta)}{2(2 \alpha-\beta)(\alpha-\beta)}$ & $\frac{1}{2}\left(o+\frac{d}{\alpha-\beta}\right)$ \\
$\mu_{i}$ & $\frac{\alpha(d-o(\alpha-\beta))}{2\left(2 \alpha^{2}-3 \alpha \beta+\beta^{2}\right)}$ & $\frac{d-o \alpha+o \beta}{2 \alpha-2 \beta}$ \\
$D_{i}$ & $\frac{\alpha(d+o(-\alpha+\beta))}{4 \alpha-2 \beta}$ & $\frac{1}{2}(d+o(-\alpha+\beta))$ \\
$\rho_{F_{i}}$ & $\frac{\alpha(d+o(-\alpha+\beta))^{2}}{2(-2 \alpha+\beta)^{2}}$ & - \\
$\rho_{C O}$ & $\frac{\alpha^{2}(d+o(-\alpha+\beta))^{2}}{2(\alpha-\beta)(-2 \alpha+\beta)^{2}}$ & - \\
$\rho_{S C}$ & $\frac{\alpha(3 \alpha-2 \beta)(d+o(-\alpha+\beta))^{2}}{2(\alpha-\beta)(-2 \alpha+\beta)^{2}}$ & $\frac{(d+o(-\alpha+\beta))^{2}}{2(\alpha-\beta)}$
\end{tabular}

\section{Discussion}

We analyze the system manner in this section through algebraic analysis.

Proposition 1. The price values in two scenarios are in the following order: $p_{i}^{\text {Dec }}>$ $p_{i}^{\text {Int }}$

Proof. $\frac{d(3 \alpha-2 \beta)+\alpha o(\alpha-\beta)}{2(2 \alpha-\beta)(\alpha-\beta)}>\frac{1}{2}\left(o+\frac{d}{\alpha-\beta}\right) \Rightarrow 0<\frac{d-o \alpha+o \beta}{4 \alpha-2 \beta}$

We know that $o \leq \frac{d}{\alpha-\beta}$ and $\alpha>\beta$, so $0<\frac{d-o \alpha+o \beta}{4 \alpha-2 \beta}$ is always true

Unlike the integrated supply chain, each disposal facility in a decentralized chain is adding its own profit to the service operation cost, and the contractor is adding its own profit $\mu_{i}$ as well. Adding the marginal profit by all chain players increases the final service price of the decentralized chain versus the integrated chain.

Proposition 2. The contractor's profit is in the following order: $\mu_{i}^{D e c}<\mu_{i}^{\text {Int }}$

Proof.

$\frac{\alpha(d-o(\alpha-\beta))}{2\left(2 \alpha^{2}-3 \alpha \beta+\beta^{2}\right)}<\frac{d-o \alpha+o \beta}{2 \alpha-2 \beta} \Rightarrow o(\alpha-\beta)^{3}<d(\alpha-\beta)^{2} \Rightarrow 0<d-o(\alpha-\beta)$ 
There is no disposal profit for individual disposal facilities in the integrated scenario, and all of supply chain profit comes from the contractor's profit. Essentially, contractor should divide the profit among the players of the supply chain. Without having the disposal facility profit $\left(h_{i}\right)$ in the integrated chain, contractor has more space for increasing its profit margin.

Proposition 3. The service demand for each facility in two scenarios are in the following order: $D_{i}^{\text {Dec }}<D_{i}^{\text {Int }}$

Proof. $\frac{\alpha(d+o(-\alpha+\beta))}{4 \alpha-2 \beta}<\frac{1}{2}(d+o(-\alpha+\beta)) \Rightarrow \frac{\alpha}{2 \alpha-\beta}<1 \Rightarrow \beta<\alpha$

Based on proposition 1, price in the decentralized chain is higher than the integrated chain. Therefore, the demand for the service provided by the decentralized chain would be lower than the demand for the integrated chain.

Proposition 4. The supply chain profit in coordinated scenario is more than the supply chain profit in decentralized scenario.

Proof. $\frac{(d+o(-\alpha+\beta))^{2}}{2(\alpha-\beta)}-\frac{\alpha(3 \alpha-2 \beta)(d+o(-\alpha+\beta))^{2}}{2(\alpha-\beta)(-2 \alpha+\beta)^{2}}=\frac{(\alpha-\beta)(d+o(-\alpha+\beta))^{2}}{2(-2 \alpha+\beta)^{2}}>0$

It is approved in many former studies that in most of the cases integration improves the profitability of the supply chain. Proposition 4 shows that the profitability of the solid waste disposal supply chain would be increased under cooperation versus decentralized decision making.

\section{$4 \quad$ Numerical Example}

A simple numerical example shows the variations of the equilibrium values versus different $\beta$ values. We assume $d=750, \alpha=45$, and $o=5$. Based on the initial model assumption of $\alpha>\beta, \beta$ can vary from 0 to 44.9 .

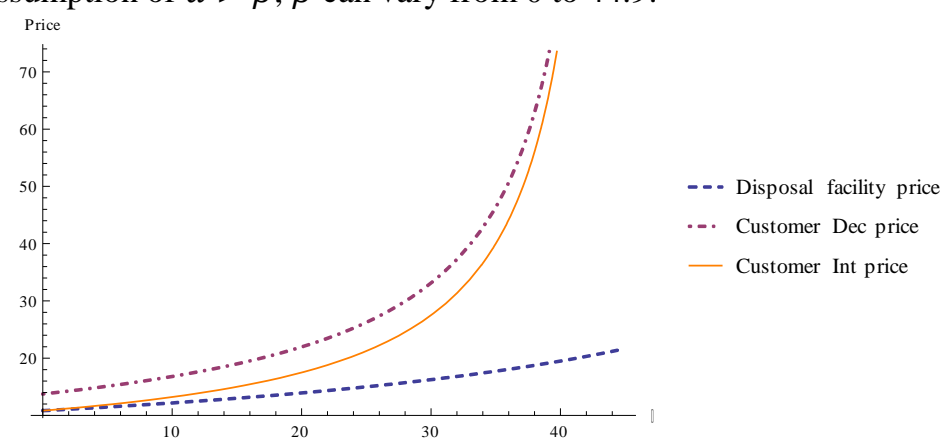

Fig. 2. Price variations versus $\beta$

Figure 2 shows that when $\beta$ gets closer to $\alpha$, disposal facility price in decentralized chain and the final price in both decentralized and integrated chains get larger. When $\beta$ gets so close to $\alpha$, it means there is not so much difference between two service providers, and the services are more substitutable. Unlike the current model, if there is not an assumption of same price for the disposal facilities, it would be expected that there would be lowering-price contest between the competitors. 


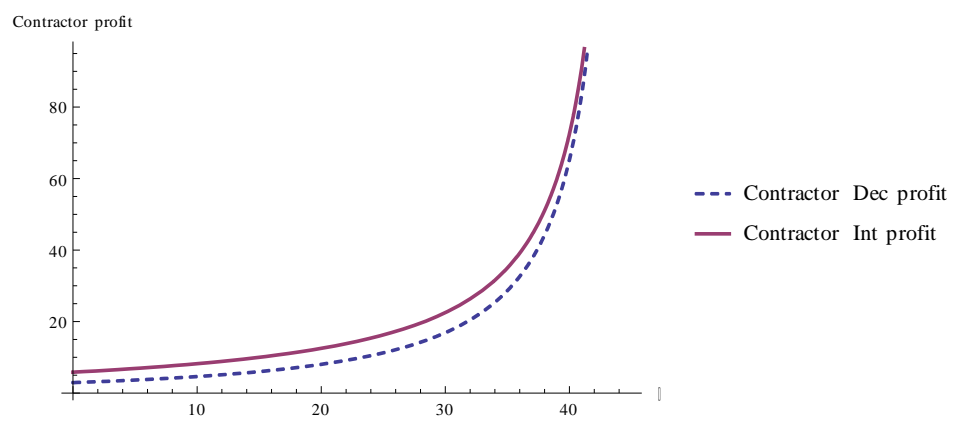

Fig. 3. Contractor profit versus $\beta$

Figure 2 noted that increasing $\beta$, increases the service price in both scenarios. By selling more expensive service, it is expected that the contractor may make more profit as well. Figure 3 shows that the contractor makes more profit in integrated channel compared to the decentralized one.

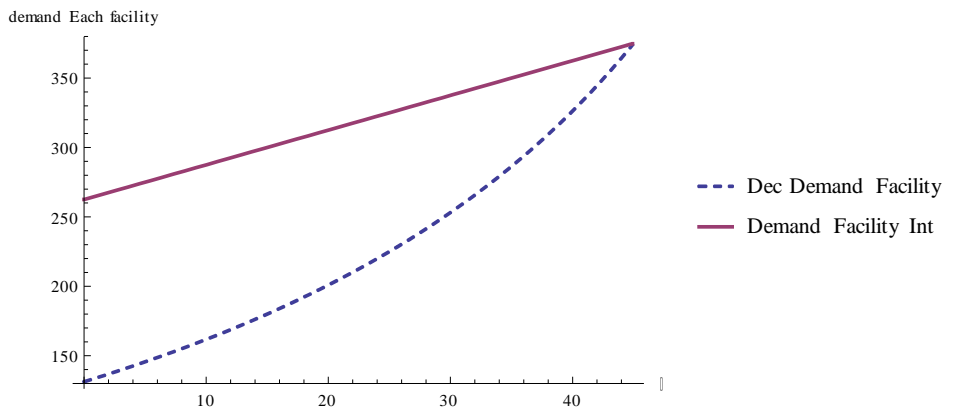

Fig. 4. Demand for each disposal facility versus $\beta$

The demand which depends on the sale price will determine the total profit in both scenarios. In Propositions 1 and 2, we proved that the integrated price is lower than the price in the decentralized scenario, and the total profit for the integrated scenario is greater than the decentralized one. Thus, we conclude that the demand level is higher in the integrated scenario. From managerial viewpoint, if the facilities want more profit, they should follow a scenario which leads to more demand.

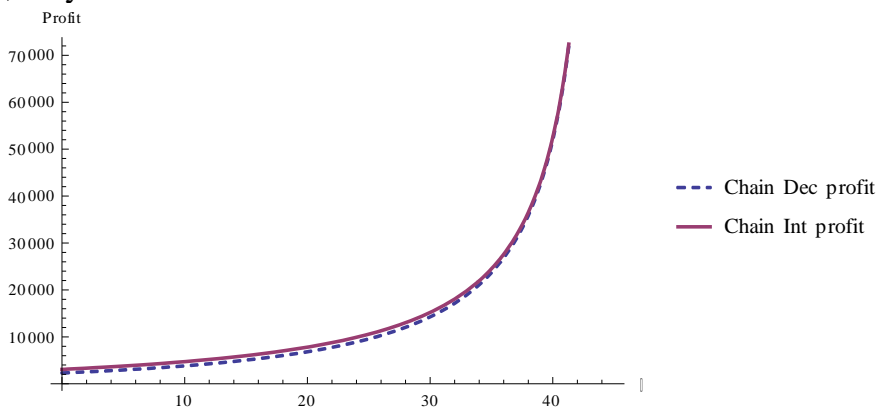

Fig. 5. Total profit versus $\beta$

Larger $\beta$ values lead to more demand and higher prices, therefore as much as two services become more substitutable, the total profit of the disposal chain would in- 
crease. Figure 5 shows that the total profit in the integrated chain is slightly more than the profit of the decentralized chain.

\section{Conclusions}

The waste management practice of a service supply chain with two disposal facilities and a contractor is studied. It is shown that working under an integrated supply chain platform can slightly improve the profitability of the chain. If the service of the disposal facilities become more similar, they can sell the service at a higher price both in the decentralized and integrated supply chains. Under the parameter assumptions of this study, the demand would be motivated if the service of both disposal facilities become more similar.

\section{References}

[1] H. Asefi, S. Lim, M. Maghrebi, and S. Shahparvari, "Mathematical modelling and heuristic approaches to the location-routing problem of a cost-effective integrated solid waste management," Ann. Oper. Res., vol. 273, no. 1-2, pp. 75-110, 2019.

[2] R. Heidari, R. Yazdanparast, and A. Jabbarzadeh, "Sustainable Design of a Municipal Solid Waste Management System Considering Waste Separators: A Real-World Application," Sustain. Cities Soc., p. 101457, 2019.

[3] M. Mohammadi, S.-L. Jämsä-Jounela, and I. Harjunkoski, “Optimal planning of municipal solid waste management systems in an integrated supply chain network," Comput. Chem. Eng., vol. 123, pp. 155-169, 2019.

[4] A. Chinasho, "Review on community based municipal solid waste management and its implication for climate change mitigation," Am. J. Sci. Ind. Res., vol. 6, no. 3, pp. 41-46, 2015.

[5] A. Pires, G. Martinho, S. Rodrigues, and M. I. Gomes, "Optimization in Waste Collection to Reach Sustainable Waste Management," in Sustainable Solid Waste Collection and Management, Springer, 2019, pp. 207-238.

[6] I. Alam, G. Alam, S. Ayub, and A. A. Siddiqui, "Assessment of Bio-medical Waste Management in Different Hospitals in Aligarh City," in Advances in Waste Management, Springer, 2019, pp. 501-510.

[7] J. A. P. de Oliveira, "Intergovernmental relations for environmental governance: Cases of solid waste management and climate change in two Malaysian States," J. Environ. Manage., vol. 233, pp. 481-488, 2019.

[8] K. V. Shah and D. D. Shah, "An Approach Towards Sustainable Municipal Solid Waste Management in India," in Waste Management and Resource Efficiency, Springer, 2019, pp. 1067-1076.

[9] V. Linderhof, P. Kooreman, M. Allers, and D. Wiersma, "Weight-based pricing in the collection of household waste: the Oostzaan case," Resour. Energy Econ., vol. 23, no. 4, pp. 359-371, 2001.

[10] P. J. Van Beukering, H. Bartelings, V. G. Linderhof, and F. H. Oosterhuis, "Effectiveness of unit-based pricing of waste in the Netherlands: Applying a general equilibrium model," Waste Manag., vol. 29, no. 11, pp. 2892-2901, 2009.

[11] Z. Han, D. Zeng, Q. Li, C. Cheng, G. Shi, and Z. Mou, "Public willingness to pay and participate in domestic waste management in rural areas of China," Resour. Conserv. Recycl., vol. 140, pp. 166-174, 2019. 